\title{
OPERATIONAL ANALYSIS SUPPORT TO DEFENCE IN NORWAY
}

\author{
Jan Erik TORP
}

Most of the military operational analysis (OA) in Norway takes place at the Norwegian Defence Research Establishment (FFI). FFI was established in 1946 by initiative of scientists that had participated in military research teams in the United Kingdom, during World War II. No Norwegians did, however, participate in operational analysis units in the UK at that time. OA was therefore not carried out at FFI in its earliest days, but a small OA unit—named the Systems Group — was established in the late 50 's.

The systems group grew steadily over the years, and in 1985 the Division for Systems Analysis was established, with about 20 scientists. The division continued to grow during the late 80 's and early 90 's, and in 2003 it numbered around 45 scientists. In 2004 a complete reorganisation of FFI was carried out, and the new Analysis Division in practice represented a continuation of the Division for Systems Analysis. It is a trend, however, that more OA is performed within other divisions at FFI, while not all the work in the Analysis Division is OA. Generally, the Analysis Division carries out higher level analyses, while OA in support of acquisitions, etc. are carried out at other divisions. The Analysis Division today has about 55 scientists.

OA can be defined in several ways, and there is not one universally agreed definition. Definitions of OA also tend to be very general, and make it hard to separate OA from "non-OA" analyses. In a wider interpretation of OA, it could be argued that many analyses carried out in the MoD, in military schools, in various staffs in the military organisation, in the industry and by consultants are also military OA. Using a more narrow interpretation, it could be argued that almost all military OA in Norway today is performed by FFI. In the following, the latter will be assumed, and only OA activities at FFI will be described.

FFI is an independent department under the Norwegian Ministry of Defence. It is not an integrated part of the $\mathrm{MoD}$, and neither is it a part of the military organisation. 
FFI's Director General reports to a board that is appointed by the MoD. All employees at FFI are civil servants of the Norwegian government. FFI is, however, required to be funded by selling projects to the MoD and other parts of the military organisation. It is also allowed to sell projects to other parts of the government, or even to private companies, but the policy is that this should only be done if such projects are consistent with other research at FFI, and its priority areas.

All the work at FFI is project organised. As mentioned, all income to FFI is earned by selling projects with a clearly defined aim, scope and size. All scientists are assigned to one or more projects. Consequently, the relevant projects will have to pay for the salaries of the scientists. Some support personnel that serves all of FFI receive their salary from money taken from the research projects as overhead. The same applies also to other operating cost that can not be attributed to one single project.

FFI has today about 650 employees. Approximately 450 of these are scientists. In 2006, the turnover of FFI was about 70 million Euros. Approximately $31 \%$ of this comes from a "block funding" from the MoD. In practice, this is money where FFI itself to a large extent decides how thuc money are used (but all of them are used for funding research projects). The Analysis Division had a turnover of approximately 8 million Euros in 2006. About 13\% of the income of FFI in 2006 came from outside the MoD or military organisations. The percentage is about the same for the Analysis Division.

Work in the Analysis Division includes:

- $\quad$ Long Term Defence planning

- Defence Economy

- Logistics studies

- Analysis Support to Acquisition Processes

- Security Policy Studies

- Critical Infrastructure Protection.

Scientists at the Analysis Division have a background from political sciences, economy and science and technology. In Norway, there is no formal education for operational analyses, although individual courses at some of the universities are relevant. In practice, scientists with a background from sciences (mathematics, physics, informatics, statistics, economy, etc) have to be given "on the job training" in OA.

Being the only place in Norway where military OA is carried out, international collaboration is important in order to be inspired by work performed by others, take part in discussions, engage in collaborative projects, etc. The NATO RTO/SAS-panel, and the activities under the panel, is maybe the most important forum for international 
collaboration. Of great benefit are also the trilateral collaboration between UK (Dstl), the Netherlands (TNO) and Norway (FFI), and bilateral relations with Sweden (FOI) and France (DGA/CAD). On a more ad-hoc basis, FFI (Analysis Division) has also had contact with Finland, Canada, Germany, Poland, Latvia, Serbia and Bulgaria. Participation in the wider international OR community (trough conferences, workshops, etc) is also valuable. 